\title{
The structure of CYP101D2 unveils a potential path for substrate entry into the active site
}

\author{
Wen Yang ${ }^{*}$, Stephen G. Bell ${ }^{\dagger, 1}$, Hui Wang*, Weihong Zhou*, \\ Mark Bartlam ${ }^{*}, 1$, Luet-Lok Wong ${ }^{\dagger}$, Zihe Rao $^{*}, \ddagger$
}

* Tianjin Key Laboratory of Protein Science, College of Life Sciences, Nankai University, Tianjin 300071, China

$\uparrow$ Department of Chemistry, University of Oxford, Inorganic Chemistry Laboratory, South Parks Road, Oxford OX1 3QR, UK

† Laboratory of Structural Biology, Tsinghua University, Beijing, 100084, China

${ }^{1}$ Address correspondence to: Stephen G. Bell, Tel: +44 01865 272679; Fax: +44 (0)1865 272690; E-mail: stephen.bell@,chem.ox.ac.uk; or Mark Bartlam, Tel/Fax: +86 22 23502351; E-mail: bartlam@,nankai.edu.cn.

CYP101D2 from Novosphingobium aromaticivorans DSM12444 is closely related to CYP101D1 from the same bacterium and to P450cam (CYP101A1) from Pseudomonas putida. All three are capable of oxidising camphor stereoselectively to 5-exohydroxycamphor. The crystal structure of CYP101D2 reveals that the likely ferredoxin binding site on the proximal face is largely positively charged, similar to that of CYP101D1. However, both the native and camphor-soaked forms of CYP101D2 have open conformations with an access channel. In the active site of the camphor-soaked form the camphor carbonyl interacts with the heme iron-bound water. Two other potential camphor binding sites were also identified from electron densities in the camphor-soaked structure: one is located in the access channel, flanked by the $\mathrm{B} / \mathrm{C}$ and $\mathrm{F} / \mathrm{G}$ loops and the I helix, and the other in a cavity on the surface of the enzyme near the $F$ helix side of the F/G loop. The observed open structures may be conformers of the CYP101D2 enzyme which enable the substrate to enter the buried active site via a conformational selection mechanism. The second and third binding sites may be intermediate locations of substrate entry and translocation into the active site, and provide insight into a multi-step substrate binding mechanism.

\section{Short title: Crystal structure of CYP101D2}

Keywords: Novosphingobium aromaticivorans DSM12444; cytochrome P450; crystal structure; open conformation; access channel; multi-step binding mechanism 


\section{Introduction}

The cytochrome P450 (CYP) superfamily of heme-dependent monooxygenases is widely distributed and catalyses $\mathrm{C}-\mathrm{H}$ bond oxidation in a broad range of organic molecules [1, 2]. Through this chemically remarkable reaction and other activities CYP enzymes are involved in a variety of biochemical processes including biosynthesis, biodegradation and xenobiotic metabolism [3, 4]. Despite low sequence similarities, structurally characterised CYP enzymes have a similar overall fold, but considerable structural diversity within this fold leads to different substrate specificity [5]. The structures of the substrate-free and substrate-bound forms of CYP enzymes often have similar closed or open conformations with little or no change upon substrate binding. CYP enzyme access channels have been classified via a systematic analysis of structurally characterised enzymes [6]. Substrate entry and binding are mainly controlled by residues within the $\mathrm{B}, \mathrm{B}^{\prime}, \mathrm{F}$ and $\mathrm{G}$ helices and the $\mathrm{B} / \mathrm{C}$ and $\mathrm{F} / \mathrm{G}$ loops [68]. However, the details of how substrates are recognized and enter the active site are often poorly defined. MD simulations of camphor binding to CYP101A1 from Pseudomonas putida suggest that protein backbone conformational changes and aromatic side chain rotations occur to allow camphor entry into the active site, and experiments have shown that salt bridge interactions modulate substrate access $[7,9,10]$. Based on theoretical and NMR studies a second camphor binding site, on the protein surface of CYP101A1, and a two-step mechanism for camphor binding were proposed [11]. Multi-step binding mechanisms and multiple substrate occupancy have been investigated in the mammalian enzymes CYP3A4 [12] and CYP1A2 [13].

Crystal structures of different substrate-bound forms of CYP119 from Sulfolobus acidocalderius show significant conformational dissimilarities [14-16], and NMR studies indicate that multiple conformations of the substrate-free enzyme may exist $[17,18]$. This may allow substrate binding to occur via a process of conformation selection, as suggested from kinetic analysis of substrate binding to P450 EryK (CYP113A1) from Saccharopolyspora erythraea [19]. Crystal structures of many forms of CYP101A1 have been determined in order to better understand $\mathrm{P} 450$ catalysis. These include the substrate and product complexes [20,21], the ferrous-CO and oxygen bound forms [22-24], and mutant enzyme structures $[25,26]$. In the majority of these, a closed conformation is observed and structural information on substrate entry into the active site is not available. Open conformations of CYP101A1 have been induced by binding adamantyl substrates linked to dansyl fluorophores or ruthenium complexes that force open the enzyme structure [27-29]. Recently, substrate-free structures of CYP101A1 have been reported in an open conformation similar to those observed with the linker substrates [30].

The CYP101A1 homologues, CYP101D2 and CYP101D1, from Novosphingobium aromaticivorans DSM12444 both bind camphor and oxidise it stereoselectively to 5-exohydroxycamphor with high activity and coupling of product formation to NADH consumption. Both enzymes utilise a class I electron transfer system consisting of a NADHdependent, FAD-containing ferredoxin reductase, ArR, and a [2Fe-2S] ferredoxin, Arx, which are analogous to putidaredoxin reductase $(\mathrm{PdR})$ and putidaredoxin $(\mathrm{Pdx})$ of the CYP101A1 system. We have previously solved the crystal structures of ArR, Arx and CYP101D1, a complete physiological class I electron transfer system [31]. The structures and the kinetic data indicate that electrostatic interactions are important in protein-protein recognition. Here we report the structure of CYP101D2 in the native and camphor-soaked forms. Both structures have an open conformation with an access channel. The camphor-soaked structure contains three regions of electron density that could be modelled as camphor molecules. One putative camphor molecule is located in the access channel close to the $\mathrm{F} / \mathrm{G}$ and $\mathrm{B} / \mathrm{C}$ loops while the second is found at the enzyme surface on the F helix side of the F/G loop. The third 
camphor molecule is bound within the active site where its carbonyl oxygen interacts with the water ligand of the heme iron. 


\section{Experimental}

\section{Cloning, expression and purification}

An N-terminal $6 \times$ His tag was incorporated into CYP101D2 by transferring the encoding gene into the expression vector pET28a(+) (Novagen Inc.) using the Nde I and Hind III restriction enzymes [32]. The recombinant plasmid was transformed into Escherichia coli BL21(DE3). A single colony was cultured at $310 \mathrm{~K}$ in LB medium containing $50 \mu \mathrm{g} \mathrm{mL} \mathrm{L}^{-1}$ kanamycin, to an $\mathrm{OD}_{600}$ of $0.6-0.8$, and then induced with $0.5 \mathrm{mM}$ IPTG (isopropyl- $\beta$-Dthiogalactopyranoside) for $18 \mathrm{~h}$ at $293 \mathrm{~K}$. The cells were harvested by centrifugation, resuspended in $1 \times$ phosphate buffered saline (PBS) at $\mathrm{pH} 7.4$ and then disrupted by sonication at $277 \mathrm{~K}$. The supernatant obtained by centrifugation at $27,000 \mathrm{~g}$ for $30 \mathrm{~min}$ was loaded onto a $\mathrm{Ni}^{2+}$-chelating affinity column pre-equilibrated with $1 \times$ PBS buffer. After washing with 200 $\mathrm{mL}$ of $1 \times$ PBS, $\mathrm{pH} 7.4,20 \mathrm{mM}$ imidazole, the target protein was eluted from the column with $1 \times$ PBS, pH 7.4, $300 \mathrm{mM}$ imidazole. The protein was concentrated and then applied to a Superdex-200 (GE Healthcare) column equilibrated with $1 \times$ PBS buffer. The red coloured fractions were collected and concentrated in buffer A (20 mM Tris, $\mathrm{pH}$ 8.0, $1 \mathrm{mM}$ DTT). The protein was further purified using a Resource Q anion-exchange column (GE Healthcare), eluting with a linear gradient of $0-1 \mathrm{M} \mathrm{NaCl}$ in buffer A. The purity of CYP101D2 was estimated to be $>95 \%$ by SDS-PAGE analysis (Fig. S1).

\section{Crystallisation and substrate soaking experiments}

The purified CYP101D2 protein was concentrated to $50 \mathrm{mg} \mathrm{mL}^{-1}$ in crystallisation buffer (20 $\mathrm{mM}$ Tris, $\mathrm{pH} 8.0,150 \mathrm{mM} \mathrm{KCl}$ ). Crystallisation was performed by the hanging-drop vapourdiffusion method at $291 \mathrm{~K}$. The protein solution $(1 \mu \mathrm{L})$ was mixed with an equal volume of reservoir solution and equilibrated with $200 \mu \mathrm{L}$ reservoir solution. Crystal screening was carried out with Hampton Research Crystal Screen kits. Initially, small crystals were obtained using 0.1 M HEPES, pH 7.5, 2\% v/v PEG 400, $2.0 \mathrm{M}$ ammonium sulfate (condition No.39 of Crystal Screen I) and further optimisation was performed by adjusting the buffer, $\mathrm{pH}$ and concentration of precipitant. Good quality crystals were obtained after about one week from $0.1 \mathrm{M}$ Tris, $\mathrm{pH} 8.3,2.1 \mathrm{M}$ ammonium sulphate and 4\% v/v PEG 400. The camphor substrate was soaked into crystals of native CYP101D2 by adding solid camphor to the drops containing the crystals. Camphor saturated the crystal solution after several days and the soaking time was varied from one week to one month.

\section{Data collection and structure determination}

Immediately prior to data collection all crystals were cryoprotected by the addition of $20 \%$ v/v glycerol. X-ray diffraction data were collected in-house at $100 \mathrm{~K}$ on a Mar345 image plate using $\mathrm{Cu} \mathrm{K \alpha}$ radiation $(\lambda=1.5418 \AA)$ from a Rigaku MicroMax-007 rotating anode X-ray generator operating at $40 \mathrm{kV}$ and $20 \mathrm{~mA}$. All diffraction data were indexed, integrated and scaled with the HKL2000 package [33].

The structure of native CYP101D2 was solved by molecular replacement (MR) methods using the program Phaser [34] in the CCP4 suite [35] with CYP101D1 as a search model (PDB code: 3LXH). The initial model was then rebuilt using Coot [36] and refined by Refmac5 [37]. The CYP101D2 camphor-soaked structure was solved by MR methods using the native CYP101D2 as a search model. The programs Coot and Refmac5 were used for manual adjustment and refinement of the model.

The stereochemical quality of the structures was examined with the program PROCHECK [38]. The data collection and refinement statistics are summarised in Table 1. The coordinates for the crystal structures of native CYP101D2 and camphor-soaked CYP101D2 have been deposited in the Protein Data Bank with the accession codes 3NV5 and 3NV6, respectively. 


\section{Results}

\section{Structure of CYP101D2}

The crystal structures of native and camphor-soaked CYP101D2 were solved at $2.4 \AA$ and 2.2 $\AA$ resolution respectively. The final models of both structures contained a single CYP101D2 molecule. The native structure contained a single diethylene glycol molecule $\left(\mathrm{C}_{4} \mathrm{H}_{10} \mathrm{O}_{3}, \mathrm{DEG}\right)$ and was traced from residues 10-413 (out of 416) with the exception of residues 92 and 93 in the $\mathrm{B}^{\prime}$ helix and residues 189-191 in the F/G loop. The camphor-soaked structure was also traced from residues 10-413, and the entire $\mathrm{B}^{\prime}$ helix and F/G loop could be modelled.

CYP101D2 has the characteristic trigonal prism-shaped, mixed $\alpha / \beta$ structure commonly found for CYP enzymes (Fig. 1a). The organisation of the secondary structure elements of native and camphor-soaked CYP101D2 are virtually identical (r.m.s.d: $0.49 \AA$ for $399 \mathrm{C} \alpha$ atoms), and both are similar to CYP101D1 (PDB code: $3 \mathrm{LXH}$ ) and CYP101A1 (PDB code: 2CPP; Fig. S2 and Table S1). However, both structures have an open conformation resulting from shifts of the F helix (residues 173-188), G helix (192-217), H helix (225-232) and the N-terminus of the I helix (241-261). These helices are pushed away from the heme, thus opening an access channel from the heme to the enzyme surface (Fig. 1b-d and Fig. S2c). These arrangements resemble the open conformations found in the substrate-free (PDB code: 3L61)[30] and linker substrate-bound CYP101A1 structures (Ru-F8bp-Ad, PDB code: 1K2O[28]; D-4-Ad, PDB code:1RF9; D-8-Ad, PDB code: 1RE9[29]) (Fig. 1d and Fig. S2).

The structure of native CYP101D2 contains electron density in the active site that has been modelled as DEG, which is present in the PEG 400 from the crystallization buffer. The carbon atoms of the DEG molecule are in van der Waals contact with Leu250, Gly254, Thr258 and Val301 (Fig. S3), while the $\mathrm{O} 2$ and $\mathrm{O} 3$ atoms are hydrogen bonded to Wat568 (3.1 $\AA$ ), the sixth ligand to the heme iron (Fe-O 2.2 $\AA$ ), and Glu303 (3.3 $\AA$ ), respectively.

Analysis of the crystal packing interactions of CYP101D2 revealed that the $F$ and $G$ helices and F/G loop have extensive interactions with neighbouring molecules (Fig S4). This is also the case for the open CYP101A1 structure (PDB code: 3L61). Such crystal packing interactions may help maintain the open conformation observed in these crystal structures.

\section{Structure of camphor-soaked CYP101D2}

Co-crystallisation experiments with CYP101D2 and different camphor concentrations were carried out $>500$ conditions. CYP101D2 always crystallised in an open conformation similar to that described above for the native structure rather than a closed conformation that might be expected. The open camphor-soaked CYP101D2 structure shows three regions of difference in the electron density map, compared to the native form, consistent with the presence of bound camphor molecules. One camphor molecule is located in the active site, the second in the access channel, and the third in a groove on the enzyme surface (Fig. 2a). The electron densities of the second and third camphor molecules are weaker than the active site camphor, presumably due to greater disorder and lower occupancy as the substrate becomes more exposed to the external solvent.

The camphor closest to the enzyme surface is located 15-16 $\AA$ from the heme iron, in a cavity on the $F$ helix side of the F/G loop that is defined by the residues Glu156, Pro159, Val160, Ser178, Ala181, Arg182, Thr185, Arg186, Leu256, Asp257 and Val260 (Fig. 2a,b, Fig. S5, Fig. S6 and Table S2). The electron density difference map revealed a positive peak $(5.7 \sigma)$ the size and shape of which is consistent with a camphor molecule; the contacts with surrounding residues are all within 3.5-4 $\AA$ and only solid camphor was added to the soaking drop. After refinement of the camphor-soaked CYP101D2 structure, the average B-factor of this camphor is $\sim 67.6 \AA^{2}$. In the native structure only a single water molecule can be identified in this cavity with a hydrogen bond to the carbonyl oxygen of Ser178 (3.2 $\AA$ ). No camphor has been detected in this cavity in the crystal structures of CYP101A1 or CYP101D1 which are instead filled with several water molecules. However, a camphor 
molecule has been computationally docked in this cavity in CYP101A1, and a binding constant of $43 \mu \mathrm{M}$ has been calculated for a second camphor molecule using NMR techniques ( $\sim 16 \AA$ from the heme iron but of unknown location although the distance consistent with the position of this surface cavity)[11].

The second camphor molecule in CYP101D2 is located above the active site, in the access channel and approximately $12 \AA$ from the heme iron (Fig. 2a,c). The peak intensity in the difference map is similar to that located in the cavity on the enzyme surface $(5.8 \sigma)$. Again, this electron density could be assigned to a camphor molecule despite being relatively poor, which may be the result of partial occupancy and multiple binding conformations due to weak interactions with the residues lining the access channel. Following refinement of the camphor-soaked CYP101D2 structure, the average B-factor of this second camphor is $\sim 67.6$ $\AA^{2}$. Residues Phe87, Tyr96, Met98, Met184, Thr185, Leu199, Leu253 and Ile401 are in contact with this camphor molecule (Fig. 2c, Fig. S6 and Table S3), and the camphor carbonyl oxygen atom could interact with water molecules or the backbone carbonyls of Met184 and Thr185. Further analysis on the possible orientations of these two camphor molecules is provided in the supplementary information (Fig. S6).

The third camphor molecule was unambiguously modelled into difference electron density with a strong positive peak of $8.9 \sigma$ (the average B-factor of this camphor is $\sim 40.4$ $\AA^{2}$ ). The camphor is bound within the active site, and the centre of the molecule is positioned approximately $7 \AA$ from the heme iron (Fig. 2d). Residues Ile86, Met98, Thr101, Leu250, Leu253, Gly254, Thr258, Val301, Ile401 and Val402 are in van der Waals contact with the camphor carbon atoms (Fig. 2d, Fig. S7, Table 2 and Table S1). The camphor carbonyl oxygen forms a hydrogen bond $(2.8 \AA)$ with Wat589, the sixth ligand of the heme iron. The closest camphor carbon to the iron atom is $\mathrm{C} 10$ ( $4.9 \AA)$, while the camphor carbonyl oxygen is $4.4 \AA$ away. Although the selectivity of camphor oxidation by CYP101D2 is $\geq 99 \%$ for 5 exo-hydroxycamphor, the $\mathrm{C} 5$ carbon of camphor is $7.5 \AA$ away from the heme iron.

\section{The access channel and active site}

The access channel is $20 \AA$ deep and flanked by residues Tyr29, Phe87, Pro89, Tyr96, Met184, Thr185, Arg186, Pro187, Met195, Leu199, Asn203, Asp257, Val401 and Val402 (Fig. S3a and Table S3). The side chains of Met184, Thr185 and Leu199 are pushed 5.5-6.0 $\AA$ away from the active site compared to the equivalent residues in the closed CYP101D1 and CYP101A1 structures, where they form a cap over the substrate (Fig. S8). In both CYP101D2 structures, the side chain of the $\mathrm{B}^{\prime}$ loop residue, Tyr96 points away from the heme towards the solvent (Fig. 2c, Fig. S3a, and Fig. S8), while in both native and camphor-bound CYP101D1 and CYP101A1 Tyr96 points inwards at the heme, and the side chain forms a hydrogen bond with the camphor carbonyl. Notably, the Tyr96 side chain moves by $\sim 5 \AA$ towards the entrance of the access channel in the camphor-soaked CYP101D2 structure, presumably to accommodate the camphor molecule that is located in the access channel but not yet inside the active site (Fig. 2c). The side chain of Phe 87 also shifts significantly and flips by $\sim 90^{\circ}$ between native and camphor-soaked CYP101D2 structures (Fig. 2c). The electron density of Phe87 in the camphor-soaked structure is less well defined compared to the native structure. The lower resolution of the Phe87 side chain and the second camphor molecule in this structure may be due to different camphor occupancy and binding orientations resulting in different Phe 87 orientations. Even though the concentration of camphor and the soaking times were varied electron density maps obtained for the camphor molecule and Phe87 side chain were always similar.

In the camphor-soaked CYP101D2 structure, Asp257 forms a salt bridge with Arg186 but in the native structure this salt bridge is broken and the Arg 186 side chain moves towards the bulk solvent and forms a new salt bridge with Glu156 (Fig. 2b and Fig. S9). The backbone carbonyl oxygen of Asp257 in both CYP101D2 structures interacts with the side-chain 
nitrogen of Asn261 (Fig. 3a). This Asp-Asn interaction and the resultant rotation of the Asp carbonyl backbone is also found in CYP101D1 and in the ferrous deoxy form of CYP101A1 [24]. The I helix groove of CYP101D2 shifts slightly towards the N-terminus compared to its position in closed CYP101A1 and CYP101D1 structures, mirroring the shift of this groove in the open conformations of CYP101A1 (Fig. S10) [29]. These movements in CYP101D2 result in a shift of the side chain of Thr258 to a similar orientation observed for Thr252 in the ferrous-oxy form of CYP101A1, but different to those seen in the closed structures of CYP101A1 and CYP101D1.

A hydrogen-bonded network of four water molecules is located in the vicinity of the heme propionate groups of the native CYP101D2 structure (Fig. 3b,c). In the camphor-soaked structure there are fewer water molecules in this region and the Glu303 side chain and the Aring propionate group of the heme occupy different positions compared to the native structure (Fig. 3c). Several water molecules are also located in the access channel of both structures of CYP101D2 (Fig. S11).

\section{Proximal face}

The heme proximal surface of CYP101D2 has a preponderance of positively charged residues, including Arg112, Arg125, Arg289, Arg349, Arg362 and Arg370, that are conserved spatially and in sequence in CYP101D1 (Fig. 3d and Fig. S12). By comparison, the proximal face of CYP101A1 is less positively charged. The ferredoxin gene, Arx, is genomically associated with the CYP101D2 gene of N. aromaticivorans, and together ArR and Arx support the monooxygenase activity of CYP101D2, CYP101D1 ( $k_{\text {cat }} 39$ and $41 \mathrm{~s}^{-1}$, respectively) and three other $N$. aromaticivorans CYP enzymes [31]. These basic residues in CYP101D2 and CYP101D1 likely interact with acidic surface residues on Arx in the competent electron transfer complex (Fig. S12). Electrostatic interactions are important in Arx/CYP101D2 electron transfer, and the charge distributions are consistent with the low cross-reactivity with the CYP101 systems when the physiological ferredoxins are exchanged $[31,32,39]$. 


\section{Discussion}

CYP101D2 has a primary sequence identity of $62 \%$ with CYP101D1 and of 44\% with CYP101A1 (Fig. S13 and Table S1). However, due to crystal packing interactions the native and camphor-soaked structures of CYP101D2 have an open conformation with a clearly defined access channel, in contrast to the closed structures observed with CYP101D1 [31]. The helices of the native structure of CYP101D2 overlay closely with those of the open forms of CYP101A1. Unexpectedly the carbonyl oxygen of the active site camphor is hydrogenbonded to the heme iron-bound water rather than the Tyr96 side chain as observed in the closed structures of camphor-bound CYP101A1 and CYP101D1 (Table 2). Soaking of crystals of the native open form of CYP101D2 in a camphor solution or co-crystallisation of camphor with CYP101D2 did not result in a closed form in which the camphor in the active site is completely shielded from the solvent. Camphor binding titrations with CYP101D2 show an approximately $40 \%$ shift to the high-spin form and a $K_{\mathrm{d}}$ of $3.1 \mu \mathrm{M}$ (Fig. S14), similar to that observed for CYP101D1 [31].

The binding orientation of the camphor molecule in the active site is not consistent with the observed product profile of almost exclusive formation of 5-exo-hydroxycamphor. In addition, it is inhibitory towards the first electron transfer step. The enzyme has to undergo a change to a closed conformation with a concomitant shift in the camphor orientation, e.g. breaking the hydrogen-bond to the axial water and rotation to form a hydrogen-bond with the Tyr96 side-chain to orient the camphor $\mathrm{C} 5$ over the heme iron. This suggests that crystal packing interactions may have effectively locked CYP101D2 in the open conformation, resisting the conversion to a closed structure expected upon substrate binding, resulting in the observed camphor binding orientation. In solution, conformational dynamics of the enzyme may result in rapid transition to the closed form perhaps, even during camphor entry into the active site, initially leading to different transient binding orientations of the camphor molecule before the enzyme-substrate complex reaches its equilibrium structure.

Multi-step binding mechanisms have been proposed for CYP3A4, CYP1A2, CYP101A1 and recently for CYP107L1 (P450 PikC) [11-13, 40, 41]. In such mechanisms the substrate binds to a recognition site on the protein surface before entering the active site. The product could also leave via the same route, though alternative routes such as via the $\mathrm{F}$ and $\mathrm{G}$ helices or the E/F loop have been suggested [6]. Binding of multiple camphor molecules to CYP101A1 has been reported from NMR and UV studies [11, 42-44]. The observation of a putative camphor molecule in a surface cavity, coupled to the presence of a second camphor in the access channel of camphor-soaked CYP101D2, may be further evidence of a multi-step binding mechanism with initial substrate recognition occurring at the enzyme surface [11]. Modeling and NMR relaxation studies led to the proposal that a camphor entry/recognition site $\left(K_{\mathrm{d}} 43 \mu \mathrm{M}\right)$ may be located in this cavity in CYP101A1 and it is of note that these experiments showed that this surface-bound camphor molecule was in a highly fluxional state [11]. The B-factors associated with the camphor molecules in the access channel and the surface cavity are similar to those observed for the solvent, while the camphor molecule in the active site has a B-factor similar to that of the protein. The less specific interactions and mobile nature of the camphor molecules located in the CYP101D2 surface cavity and access channel compared to the camphor in the active site suggest that these may be snapshots of intermediate binding positions in the substrate entrance pathway into the active site (or of product release).

Many structurally characterised CYP enzymes have closed conformations with buried active sites [20, 31]. However, clearly defined access channels are not uncommon [45, 46]. Other P450 enzymes show open conformations in the absence of a bound substrate, but adopt a more closed conformation upon substrate binding, e.g. P450 EryK [19]. Double jump substrate binding studies of P450 EryK suggest a conformational selection model for 
substrate recognition and binding [19]. Crystal structures of CYP119 reveal that the enzyme undergoes major rearrangements at the active site to accommodate the binding of different substrates and NMR studies of this enzyme tagged with the unnatural amino acid ${ }^{13} \mathrm{C}-p$ methoxyphenylalanine showed that it can exist in different conformations $[15,17,18]$. These conformers are thought to be important in substrate recognition and binding, and the equilibrium between them can be perturbed using different substrates. The open structure of CYP101D2 may be a conformer which facilitates substrate entry and product exit from the active site. The movement of Tyr96 between the open and closed conformers may assist in the recognition and binding of camphor. We note, however that CYP101D2 is probably held in this open conformation by crystal packing contacts and the camphor concentration of $\sim 8 \mathrm{mM}$ $\left(1.2 \mathrm{~g} \mathrm{~L}^{-1}\right)$ is higher than those in turnover reactions $(1 \mathrm{mM})$. Therefore we cannot rule out adventitious binding at one or both of the locations outside the active site. The substrate might enter (or the product exit) the active site that is opened up by normal thermal fluctuations of the structure via the channel without forming an intermediate state. On the other hand, no adventitious binding of camphor was observed to the surface cavity of the closed conformations of CYP101D1 under a similar camphor soaking regime.

The switch between the open and the closed forms of CYP101A1 involves breaking and formation of inter-residue salt bridges and hydrogen bonds and the rearrangement of hydrophobic interactions [29]. The presence or otherwise of these interactions, together with adventitious binding of molecules such as DEG or deliberate introduction of longer substrates such as D-4-Ad, as well as crystal packing effects, may control whether crystals with open or closed conformation are obtained. Salt bridge interactions between the F helix and F/G loop and the I helix are less extensive in CYP101D2 (and CYP101D1) compared to CYP101A1 (Table S2). A network of interactions between Lys178, Asp182, Arg186 and Asp251 in CYP101A1 has been shown to play a key role in the control of the diffusion step of camphor binding and provide a link from the I helix to the bulk solvent at the enzyme surface (Table S2) $[10,47]$. These interactions are broken in the open conformations of CYP101A1 [29, 30]. The Asp257-Arg186 salt bridge is conserved in camphor-soaked CYP101D2, but Ser178 and Arg182 replace Lys178 and Asp182. Ser178 is located in the access channel while Arg182 points towards the enzyme surface (Fig. 2b and S5b). The Asp257-Arg186 salt bridge separates the camphor molecules in the surface recognition site and in the access channel site. The breaking of this salt bridge and the movement of Arg186 to interact with Glu156, which lines the surface recognition site, could open up a short route of substrate entry or product exit between these two sites. This blocking of the proposed surface recognition site and the active site camphor by the equivalent salt bridge in CYP101A1 had been noted previously [11], and in the substrate-free open CYP101A1 structure the Arg186 residue interacts with Glu156 [30]. The structure and function of mutants with substitutions to break salt bridges will be of interest $[10,47,48]$.

The proximal faces of CYP101D2 and CYP101D1 are very similar, and more positively charged than CYP101A1, while the corresponding interaction region on the Arx ferredoxin is more negatively charged compared to Pdx [31], consistent with electrostatic interactions being important in Arx/CYP101D2 and Arx/CYP101D1 binding and recognition. However, the precise role of each of the acidic residues on Arx and the basic residues on the CYP enzymes remains to be elucidated. The ArR/Arx system also supports the monooxygenase activity of at least three other CYP enzymes from $N$. aromaticivorans (CYP101B1, CYP101C1 and CYP111A2). The crystal structures of the other P450 enzymes supported by Arx will be required for a fuller analysis, and this is the subject of ongoing investigations. 


\section{Conclusions}

CYP101D2 and CYP101D1 are analogues of CYP101A1 that can catalyse the fast, efficient and stereoselective oxidation of camphor to 5-exo-hydroxycamphor. While the structure of CYP101D1 is similar to that of CYP101A1, the camphor-soaked structure of CYP101D2 has an open conformation and reveals potential camphor binding sites at the active site, in the access channel and at the enzyme surface. This open structure may be a conformer involved in substrate recognition and binding. The camphor binding sites at the enzyme surface and in the access channel may be intermediate locations of the substrate in its progression into the active site of CYP101D2 via multi-step and conformational selection mechanisms.

\section{Acknowledgements}

We are grateful to Dr Zhiyong Lou for help in data collection and processing.

\section{Funding}

The work was supported by grant 2007CB914301 from the Ministry of Science and Technology of China Project 973 (to M. B.), the Tianjin Municipal Science and Technology Commission (grant number 08SYSYTC00200) and by the Higher Education Funding Council for England (HEFCE).

Abbreviations used: CYP, cytochrome P450; DEG, diethylene glycol; PEG, polyethylene glycol; Ru-F $-\mathrm{bp}-\mathrm{Ad}$, ruthenium tris(bipyridine)-4,4'-octafluorobiphenyladamantane; D-4-Ad, dansylbutyladamantane ; D-8-Ad, dansyloctyladamantane; r.m.s.d., root mean square deviation; Pdx, putidaredoxin; PdR, putidaredoxin reductase; Arx, a [2Fe-2S] ferredoxin from $N$. aromaticivorans; ArR, oxygenase-coupled NADH-dependent ferredoxin reductase from $N$. aromaticivorans; MR, molecular replacement; MD, molecular dynamics; HEPES, 4-(2hydroxyethyl)-1-piperazineethanesulfonic acid. 


\section{References}

1 Ortiz de Montellano, P. R., ed. (2005) Cytochrome P450: Structure, Mechanism, and Biochemistry Kluwer Academic/Plenum Press, New York

2 Sigel, A., Sigel, H. and Sigel, R. (2007) The Ubiquitous Roles of Cytochrome P450 Proteins. John Wiley \& Sons

3 Cryle, M. J., Stok, J. E. and De Voss, J. J. (2003) Reactions catalyzed by bacterial cytochromes P 450. Aust. J. Chem. 56, 749-762

4 Bernhardt, R. (2006) Cytochromes P450 as versatile biocatalysts. J. Biotechno1. 124, $128-145$

5 Denisov, I. G., Makris, T. M., Sligar, S. G. and Schlichting, I. (2005) Structure and chemistry of cytochrome P450. Chem. Rev. 105, 2253-2277

6 Cojocaru, V., Winn, P. J. and Wade, R. C. (2007) The ins and outs of cytochrome P450s. Biochim. Biophys. Acta. 1770, 390-401

7 Wade, R. C., Winn, P. J., Schlichting, I. and Sudarko. (2004) A survey of active site access channels in cytochromes P450. J. Inorg. Biochem. 98, 1175-1182

8 Li, H. and Poulos, T. L. (2004) Crystallization of cytochromes P450 and substrateenzyme interactions. Curr. Top. Med. Chem. 4, 1789-1802

9 Winn, P. J., Ludemann, S. K., Gauges, R., Lounnas, V. and Wade, R. C. (2002)

Comparison of the dynamics of substrate access channels in three cytochrome P450s reveals different opening mechanisms and a novel functional role for a buried arginine. Proc. Natl. Acad. Sci. U S A 99, 5361-5366

10 Deprez, E., Gerber, N. C., Di Primo, C., Douzou, P., Sligar, S. G. and Hui Bon Hoa, G. (1994) Electrostatic control of the substrate access channel in cytochrome P-450cam. Biochemistry 33, 14464-14468

11 Yao, H., McCullough, C. R., Costache, A. D., Pullela, P. K. and Sem, D. S. (2007) Structural evidence for a functionally relevant second camphor binding site in P450cam: model for substrate entry into a P450 active site. Proteins. 69, 125-138

12 Isin, E. M. and Guengerich, F. P. (2006) Kinetics and thermodynamics of ligand binding by cytochrome P450 3A4. J. Biol. Chem. 281, 9127-9136

13 Sohl, C. D., Isin, E. M., Eoff, R. L., Marsch, G. A., Stec, D. F. and Guengerich, F. P. (2008) Cooperativity in oxidation reactions catalyzed by cytochrome P450 1A2: highly cooperative pyrene hydroxylation and multiphasic kinetics of ligand binding. J. Biol. Chem. 283, 7293-7308

14 Yano, J. K., Koo, L. S., Schuller, D. J., Li, H., Ortiz de Montellano, P. R. and Poulos, T. L. (2000) Crystal structure of a thermophilic cytochrome P450 from the archaeon Sulfolobus solfataricus. J. Biol. Chem. 275, 31086-31092

15 Nishida, C. R. and Ortiz de Montellano, P. R. (2005) Thermophilic cytochrome P450 enzymes. Biochem. Biophys. Res. Commun. 338, 437-445

16 Park, S. Y., Yamane, K., Adachi, S., Shiro, Y., Weiss, K. E., Maves, S. A. and Sligar, S. G. (2002) Thermophilic cytochrome P450 (CYP119) from Sulfolobus solfataricus: high resolution structure and functional properties. J. Inorg. Biochem. 91, 491-501

17 Lampe, J. N., Brandman, R., Sivaramakrishnan, S. and Ortiz de Montellano, P. R. (2010) 2D NMR and All-atom molecular dynamics of cytochrome P450 CYP119 reveal hidden conformational substrates. J. Biol. Chem. 285, 9594-9603

18 Lampe, J. N., Floor, S. N., Gross, J. D., Nishida, C. R., Jiang, Y., Trnka, M. J. and Ortiz de Montellano, P. R. (2008) Ligand-induced conformational heterogeneity of cytochrome P450 CYP119 identified by 2D NMR spectroscopy with the unnatural amino acid (13)C-p-methoxyphenylalanine. J. Am. Chem. Soc. 130, 16168-16169

19 Savino, C., Montemiglio, L. C., Sciara, G., Miele, A. E., Kendrew, S. G., Jemth, P., Gianni, S. and Vallone, B. (2009) Investigating the structural plasticity of a cytochrome P450: 
three-dimensional structures of P450 EryK and binding to its physiological substrate. J. Biol. Chem. 284, 29170-29179

20 Poulos, T. L., Finzel, B. C. and Howard, A. J. (1987) High-resolution crystal structure of cytochrome P450cam. J. Mol. Biol. 195, 687-700

21 Li, H., Narasimhulu, S., Havran, L. M., Winkler, J. D. and Poulos, T. L. (1995) Crystal Structure of Cytochrome P450cam Complexed with Its Catalytic Product, 5-exoHydroxycamphor. J. Am. Chem. Soc. 117, 6297-6299

22 Raag, R. and Poulos, T. L. (1989) Crystal structure of the carbon monoxide-substratecytochrome P-450CAM ternary complex. Biochemistry 28, 7586-7592

23 Nagano, S. and Poulos, T. L. (2005) Crystallographic study on the dioxygen complex of wild-type and mutant cytochrome P450cam. Implications for the dioxygen activation mechanism. J. Biol. Chem. 280, 31659-31663

24 Schlichting, I., Berendzen, J., Chu, K., Stock, A. M., Maves, S. A., Benson, D. E., Sweet, R. M., Ringe, D., Petsko, G. A. and Sligar, S. G. (2000) The catalytic pathway of cytochrome p450cam at atomic resolution. Science 287, 1615-1622.

25 Chen, X., Christopher, A., Jones, J. P., Bell, S. G., Guo, Q., Xu, F., Rao, Z. and Wong, L. L. (2002) Crystal structure of the F87W/Y96F/V247L mutant of cytochrome P-450cam with 1,3,5-trichlorobenzene bound and further protein engineering for the oxidation of pentachlorobenzene and hexachlorobenzene. J. Biol. Chem. 277, 37519-37526

26 Vidakovic, M., Sligar, S. G., Li, H. and Poulos, T. L. (1998) Understanding the role of the essential Asp251 in cytochrome p450cam using site-directed mutagenesis, crystallography, and kinetic solvent isotope effect. Biochemistry 37, 9211-9219

27 Dmochowski, I. J., Crane, B. R., Wilker, J. J., Winkler, J. R. and Gray, H. B. (1999) Optical detection of cytochrome P450 by sensitizer-linked substrates. Proc. Natl. Acad. Sci. U S A 96, 12987-12990

28 Dunn, A. R., Dmochowski, I. J., Bilwes, A. M., Gray, H. B. and Crane, B. R. (2001) Probing the open state of cytochrome P450cam with ruthenium-linker substrates. Proc. Natl. Acad. Sci. U S A. 98, 12420-12425

29 Hays, A. M., Dunn, A. R., Chiu, R., Gray, H. B., Stout, C. D. and Goodin, D. B. (2004) Conformational states of cytochrome P450cam revealed by trapping of synthetic molecular wires. J. Mol. Biol. 344, 455-469

30 Lee, Y. T., Wilson, R. F., Rupniewski, I. and Goodin, D. B. (2010) P450cam visits an open conformation in the absence of substrate. Biochemistry 49, 3412-3419

31 Yang, W., Bell, S. G., Wang, H., Zhou, W., Hoskins, N., Dale, A., Bartlam, M., Wong, L. L. and Rao, Z. (2010) Molecular characterization of a class I P450 electron transfer system from Novosphingobium aromaticivorans DSM12444. J. Biol. Chem. 285, 2737227384

32 Bell, S. G. and Wong, L. L. (2007) P450 enzymes from the bacterium

Novosphingobium aromaticivorans. Biochem. Biophys. Res. Commun. 360, 666-672

33 Otwinowski, Z. W. M. (1997) Processing of X-ray diffraction data collected in oscillation mode. Methods Enzymol. 276, 307-326

34 McCoy, A. J., Grosse-Kunstleve, R. W., Adams, P. D., Winn, M. D., Storoni, L. C. and Read, R. J. (2007) Phaser crystallographic software. J. Appl. Crystallogr. 40, 658-674

35 Collaborative Computational Project (1994) The CCP4 suite: programs for protein crystallography. Acta Crystallogr. D Biol. Crystallogr. 50, 760-763

36 Emsley, P. and Cowtan, K. (2004) Coot: model-building tools for molecular graphics. Acta Crystallogr. D Biol. Crystallogr. 60, 2126-2132

37 Murshudov, G. N., Vagin, A. A. and Dodson, E. J. (1997) Refinement of macromolecular structures by the maximum-likelihood method. Acta Crystallogr. D Biol. Crystallogr. 53, 240-255 
38 Laskowski, R. A., Macarthur, M. W., Moss, D. S. and Thornton, J. M. (1993) PROCHECK - A program to check the stereochemical quality of protein structures. J. Appl. Crystallogr. 26, 283-291

39 Bell, S. G., Dale, A., Rees, N. H. and Wong, L. L. (2010) A cytochrome P450 class I electron transfer system from Novosphingobium aromaticivorans. Appl. Microbiol. Biotechnol. 86, 163-175

40 Isin, E. M. and Guengerich, F. P. (2008) Substrate binding to cytochromes P450. Anal. Bioanal. Chem. 392, 1019-1030

41 Li, S., Ouellet, H., Sherman, D. H. and Podust, L. M. (2009) Analysis of transient and catalytic desosamine-binding pockets in cytochrome P-450 PikC from Streptomyces venezuelae. J. Biol. Chem. 284, 5723-5730

42 Lee, H., Ortiz de Montellano, P. R. and McDermott, A. E. (1999) Deuterium magic angle spinning studies of substrates bound to cytochrome P450. Biochemistry 38, 1080810813

43 Marden, M. C. and Hoa, G. H. (1987) P-450 binding to substrates camphor and linalool versus pressure. Arch. Biochem. Biophys. 253, 100-107

44 Narasimhulu, S. and Willcox, J. K. (2001) Temperature-jump relaxation kinetics of substrate-induced spin-state transition in cytochrome P450 (comparison of the wild-type and C334A mutant P450(CAM) and P450(2B4)). Arch. Biochem. Biophys. 388, 198-206

45 Ravichandran, K. G., Boddupalli, S. S., Hasermann, C. A., Peterson, J. A. and Deisenhofer, J. (1993) Crystal structure of hemoprotein domain of P450BM-3, a prototype for microsomal P450's. Science 261, 731-736

46 Bell, S. G., Xu, F., Forward, I., Bartlam, M., Rao, Z. and Wong, L. L. (2008) Crystal structure of CYP199A2, a para-substituted benzoic acid oxidizing cytochrome P450 from Rhodopseudomonas palustris. J. Mol. Biol. 383, 561-574

47 Di Primo, C., Deprez, E., Sligar, S. G. and Hui Bon Hoa, G. (1997) Origin of the photoacoustic signal in cytochrome P-450cam: role of the Arg186-Asp251-Lys178 bifurcated salt bridge. Biochemistry 36, 112-118

48 Lounnas, V. and Wade, R. C. (1997) Exceptionally stable salt bridges in cytochrome $\mathrm{P} 450$ cam have functional roles. Biochemistry 36, 5402-5417 


\section{Table and figure legends}

Table 1. X-ray data collection and structure refinement statistics for native (DEG-bound) and camphor-soaked CYP101D2.

Table 2. Protein-camphor interactions at the active site of CYP101D2, CYP101D1 and CYP101A1. ${ }^{a}$ from reference [31]. ${ }^{b}$ from reference [20].

Figure 1. a) The secondary structure of camphor-soaked CYP101D2. The B', F and G helices are coloured in red, orange and salmon, respectively. The heme moiety is shown in yellow.

b) An overlay of the camphor-soaked form of CYP101D2 and CYP101D1 (gray) highlighting the movement of the F, G and $\mathrm{H}$ helices and the $\mathrm{N}$ terminus of the I helix. The B', F, G, H and I helices of CYP101D2 are coloured in red, orange, salmon, blue and magenta, respectively.

c) The distal surface of camphor-soaked CYP101D2. The B', F and G helices and F/G loop (in green) open up to form an access channel (black circle).

d) Overlay of camphor-soaked CYP101D2 and an open CYP101A1 conformer with an adamantyl-linked ruthenium complex (Ru-F8bp-Ad) bound (PDB: 1K20). The secondary structure of CYP101A1 is shown in gray and the Ru-F8bp-Ad molecule is shown in green. The open form of CYP101A1 (PDB: 3L61) has a similar structure to Ru-F8bp-Ad-bound CYP101A1 and CYP101D2. The positions of the F, G, H and I helices of CYP101D2 closely resemble those in both these structures; the F/G loop and the residues at the $\mathrm{N}$-terminus of the $\mathrm{G}$ helix in the open forms of CYP101A1 are bent further back.

Figure 2. a) The position of the three potential camphor electron densities relative to the active site and access channel. The electron density $\left(F_{o}-F_{c}\right.$ contoured at $\left.3 \sigma\right)$ of the two potential camphor binding sites that are located in the access channel and on the enzyme surface are shown in orange. The electron density $\left(2 F_{o}-F_{c}\right.$ contoured at $\left.1 \sigma\right)$ of the camphor molecule bound in the active site is shown in blue. The camphor and heme molecule are shown in green and yellow, respectively.

b) The structure around the camphor recognition site. The electron density $\left(F_{o}-F_{c}\right.$ contoured at $3 \sigma$ ) of the camphor is coloured in orange. The helices are shown in gray and the neighbouring residues are shown in green. Asp257 forms a salt bridge with Arg186 in the camphor-soaked structure while in the native structure this salt bridge is broken and the Arg186 side chain moves away from Asp257 towards the bulk solvent and forms a new salt bridge with Glu156 (residues Arg186 and Glu156 in the native form are coloured in cyan).

c) The access channel of camphor-soaked CYP101D2. The electron density $\left(F_{o}-F_{c}\right.$ contoured at $3 \sigma$ ) of the camphor molecule located in the access channel is shown in orange. The residues involved in camphor binding are shown in green. In the $\mathrm{B}^{\prime}$ helix the Tyr96 residue moves $5 \AA$ when compared to the native CYP101D2 structure to accommodate the camphor in the access channel. The rotation of the side chain of Phe87 and the poor density map $\left(2 F_{o^{-}}-\right.$ $F_{c}$ contoured at $1 \sigma$ ), coloured in blue, in the camphor-soaked form are also shown (residues Tyr96 and Phe87 in the native form are coloured in cyan).

d) The active site of camphor-soaked CYP101D2. The residues involved in substrate binding are shown in green and the water molecule (Wat589) that occupies the sixth heme iron coordination site and which hydrogen bonds to the camphor carbonyl is shown as a red sphere. The electron density $\left(2 F_{o}-F_{c}\right.$ contoured at $\left.1 \sigma\right)$ of the camphor molecule is shown in blue.

Figure 3. a) Overlay of the residues in the I helix between CYP101D2 (green) and CYP101A1 (salmon). The conformation of the carbonyl oxygen of Asp257 is flipped by $\sim 90^{\circ}$ towards Asn261 due to a hydrogen-bond to the side-chain nitrogen of Asn261 (2.8 $)$. Two water molecules hydrogen bond to the Asp257 side chain and are shown as red spheres. 
b) The heme environment in the native form of CYP101D2. The A-ring propionate group forms a salt bridge to Arg305. The O1 atom forms hydrogen bonds to two water molecules, Wat565 and Wat436, and Wat565 forms another hydrogen bond to Wat566. The $\mathrm{O} 2$ atom of the A-ring propionate group is hydrogen-bonded to the side-chains of Tyr75 and Glu303. The

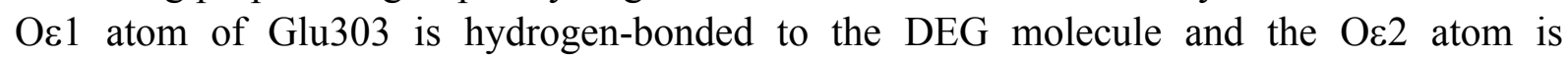
hydrogen-bonded to Wat566 and the A-ring propionate group. The heme and the relevant residues are shown in yellow and green respectively. The water molecules are shown as red spheres. The electron density $\left(2 F_{o}-F_{c}\right.$ contoured at $\left.1 \sigma\right)$ of the DEG molecule is shown in blue. c) The heme environment in the camphor-soaked structure of CYP101D2. Compared to the native structure the A-ring propionate group is rotated about $90^{\circ}$, and both the $\mathrm{O}$ atoms of Glu303 are hydrogen-bonded to the propionate group. The propionate group also forms a salt bridge to Arg305 and hydrogen bonds to two water molecules, Wat498 and Wat464. Glu303 is hydrogen bonded to Tyr75. The heme and residues are shown in yellow and green respectively. The water molecules are shown as red spheres and the electron density $\left(2 F_{o}-F_{c}\right.$ contoured at $1 \sigma$ ) of the camphor molecule is shown in blue.

d) The proximal face of CYP101D2. Negatively and positively charged surface areas are coloured in red and blue respectively. The positively charged proximal face is similar to that of CYP101D1 and more positively charged than that of CYP101A1 (Fig. S12). Highlighted are the basic residues of CYP101D2 (Arg112, Arg125, Arg289, Arg349, Arg362 and Arg370) and Ser76 which aligns with Arg77 in CYP101D1. 
Table 1.

Data collection statistics
\begin{tabular}{|l|l|l|}
\hline Data set & CYP101D2 & CYP101D2:cam \\
\hline Wavelength & 1.5418 & 1.5418 \\
\hline Space group & $\mathrm{P} 3{ }_{2} 21$ & $\mathrm{P} 3{ }_{2} 21$ \\
\hline Cell dimensions a/b/c $(\AA)$ & $86.6 / 86.6 / 124.7$ & $86.1 / 86.1 / 123.9$ \\
$\alpha / \beta / \gamma\left({ }^{\circ}\right)$ & $90.0 / 90.0 / 120.0$ & $90.0 / 90.0 / 120.0$ \\
\hline Resolution $(\AA)$ & $50-2.4$ & $50-2.2$ \\
& $(2.49-2.40)$ & $(2.28-2.20)$ \\
\hline Average $I / \sigma(I) 1$ & $25.7(3.5)$ & $33.2(5.8)$ \\
\hline Completeness $(\%)^{1}$ & $96.2(98.4)$ & $99.0(99.5)$ \\
\hline Redundancy ${ }^{1}$ & $5.4(5.1)$ & $4.7(4.4)$ \\
\hline$R_{\text {merge }}(\%)^{1,2}$ & $6.0(47.4)$ & $4.3(23.2)$ \\
\hline
\end{tabular}

Structure refinement statistics

\begin{tabular}{|l|l|l|}
\hline Resolution $(\AA)$ & $50-2.4$ & $50-2.2$ \\
\hline Average $B$-factor $\left(\AA^{2}\right)$ & 43.1 & 34.4 \\
\hline$R_{\text {work }} / R_{\text {free }}(\%)^{3}$ & $19.6 / 26.0$ & $18.9 / 25.5$ \\
\hline r.m.s.d. bond lengths $(\AA)$ & 0.014 & 0.016 \\
\hline r.m.s.d. bond angles $\left(^{\circ}\right)$ & 1.559 & 1.683 \\
\hline
\end{tabular}

Ramachandran plot

\begin{tabular}{|l|l|l|}
\hline Most favoured (\%) & 88.8 & 90.5 \\
\hline Allowed (\%) & 11.2 & 9.5 \\
\hline Generously allowed (\%) & 0 & 0 \\
\hline Disallowed (\%) & 0 & 0 \\
\hline
\end{tabular}

${ }^{1}$ Values in parentheses correspond to the highest-resolution shell.

${ }^{2} R_{\text {merge }}=\Sigma_{i}\left|I_{i}-\langle I\rangle\right| / \Sigma\langle I\rangle$, where $I_{i}$ is an individual intensity measurement and $\langle I\rangle$ is the average intensity for all the reflections.

${ }^{3} R_{\text {work }} / R_{\text {free }}=\Sigma|| F_{o}|-| F_{c}|| / \Sigma\left|F_{o}\right|$, where $F_{o}$ and $F_{c}$ are the observed and calculated structure factors, respectively. 
Table 2.

\begin{tabular}{|c|c|c|c|}
\hline Camphor atom & CYP101D2 & CYP101D1 $^{\mathrm{a}}$ & CYP101A1 $^{\mathrm{b}}$ \\
\hline $\mathrm{O}$ & Wat589(2.8 $\AA)$ & Tyr97OH (2.6 A) & 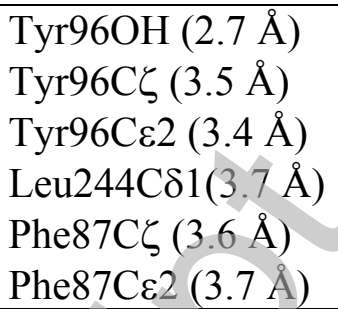 \\
\hline $\mathrm{C} 1$ & & & $\begin{array}{l}\text { Leu244C } 1(3.8 \AA) \\
\text { Tyr96OH(3.7 }\end{array}$ \\
\hline $\mathrm{C} 2$ & & $\begin{array}{l}\text { Leu251C } 81(4.0 \AA) \\
\text { Trp88CH2(4.0 } \AA)\end{array}$ & $\begin{array}{l}\text { Leu244C } 1(3.7 \AA) \\
\text { Tyr960H(3.4 } \AA)\end{array}$ \\
\hline $\mathrm{C} 3$ & $\operatorname{Met} 98 \mathrm{C} \varepsilon(4.4 \AA)$ & $\begin{array}{l}\text { Leu251C } 81(4.1 \AA) \\
\text { Thr102C } 2(4.0 \AA)\end{array}$ & \\
\hline $\mathrm{C} 5$ & $\begin{array}{l}\text { Ile86C } \delta 1(4.0 \AA) \\
\text { Thr101C } 2(4.2 \AA)\end{array}$ & $\begin{array}{l}\text { Leu251C } \delta 1(4.0 \AA) \\
\text { Gly255C }(4.0 \AA)\end{array}$ & \\
\hline C6 & $\begin{array}{l}\text { Thr101C } 22(4.3 \AA) \\
\text { Ile86C } \delta 1(4.5 \AA)\end{array}$ & $\begin{array}{l}\text { Leu251C } \delta 1(4.2 \AA) \\
\text { Gly255Ca }(3.5 \AA) \\
\text { Leu254C }(4.2 \AA)\end{array}$ & Val247C $\gamma 1(3.9 \AA)$ \\
\hline $\mathrm{C} 8$ & $\begin{array}{l}\text { Val402C } 22(3.6 \AA) \\
\text { Ile401C } 2(4.4 \AA)\end{array}$ & Val302C $\gamma 1(3.7 \AA)$ & $\begin{array}{l}\text { Val295C } 1(3.7 \AA) \\
\text { Asp297O } \delta 2(3.8 \AA)\end{array}$ \\
\hline C9 & $\begin{array}{l}\text { Ile401C } 2(3.6 \AA) \\
\text { Val301C } \gamma 1(4.4 \AA) \\
\text { Glu303C } \beta(4.1 \AA)\end{array}$ & $\begin{array}{l}\text { Val302C } 2(3.6 \AA) \\
\text { Thr259C } 2(3.6 \AA)\end{array}$ & $\begin{array}{l}\text { Val295C } 1(3.9 \AA) \\
\text { Val295C } 2(3.9 \AA)\end{array}$ \\
\hline C10 & $\begin{array}{l}\text { Thr258C } 22(4.6 \AA) \\
\text { Val301C } 1(4.2 \AA) \\
\text { Val301C } 2(4.3 \AA)\end{array}$ & $\begin{array}{l}\text { Leu2 } 254 \mathrm{C} \gamma(3.9 \AA) \\
\text { Leu254C } \delta 1(3.8 \AA) \\
\text { Thr186C } 2(3.7 \AA) \\
\text { Val403C } 2(3.9 \AA) \\
\end{array}$ & $\begin{array}{l}\text { Val247C } \gamma 1(3.6 \AA) \\
\text { Thr185C } 2(3.9 \AA)\end{array}$ \\
\hline
\end{tabular}




\section{Figures}

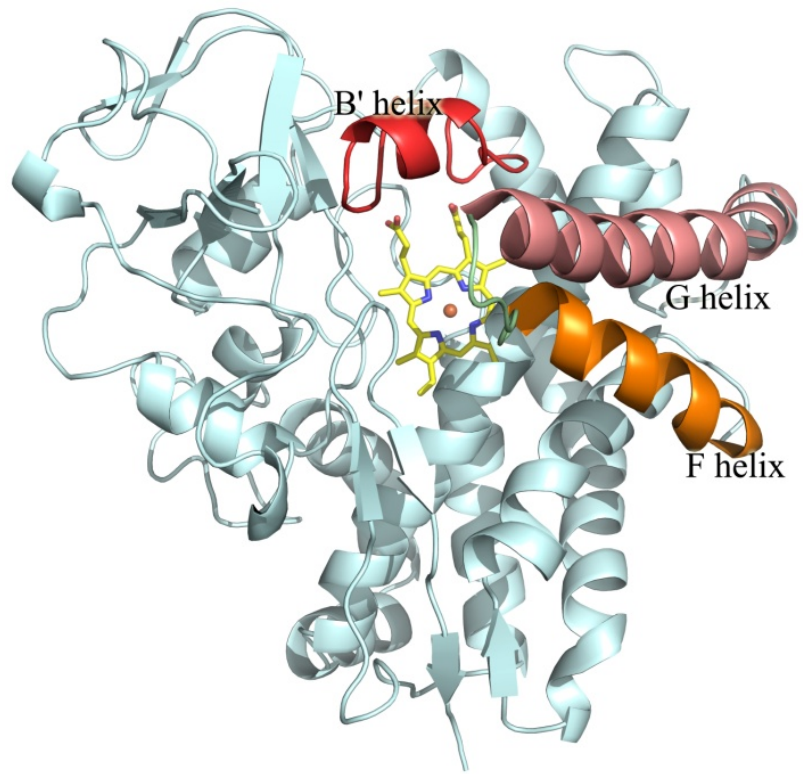

(a)

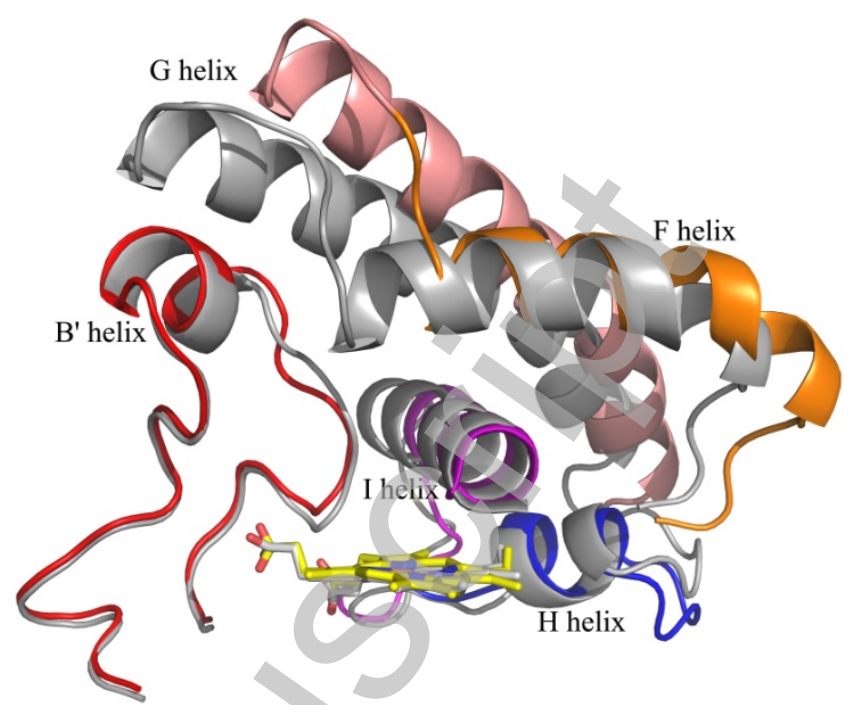

(b)

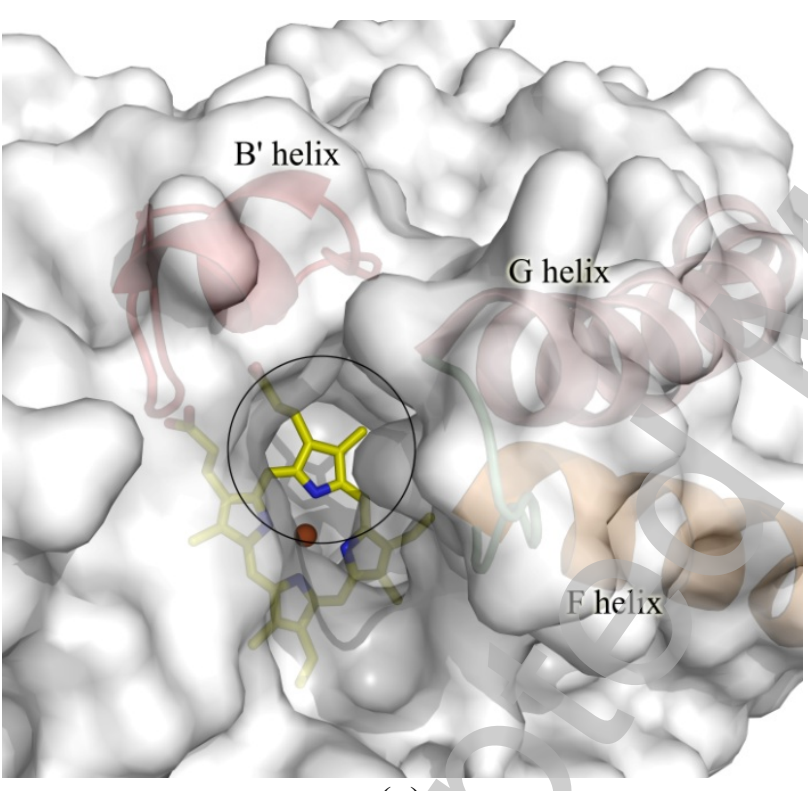

(c)

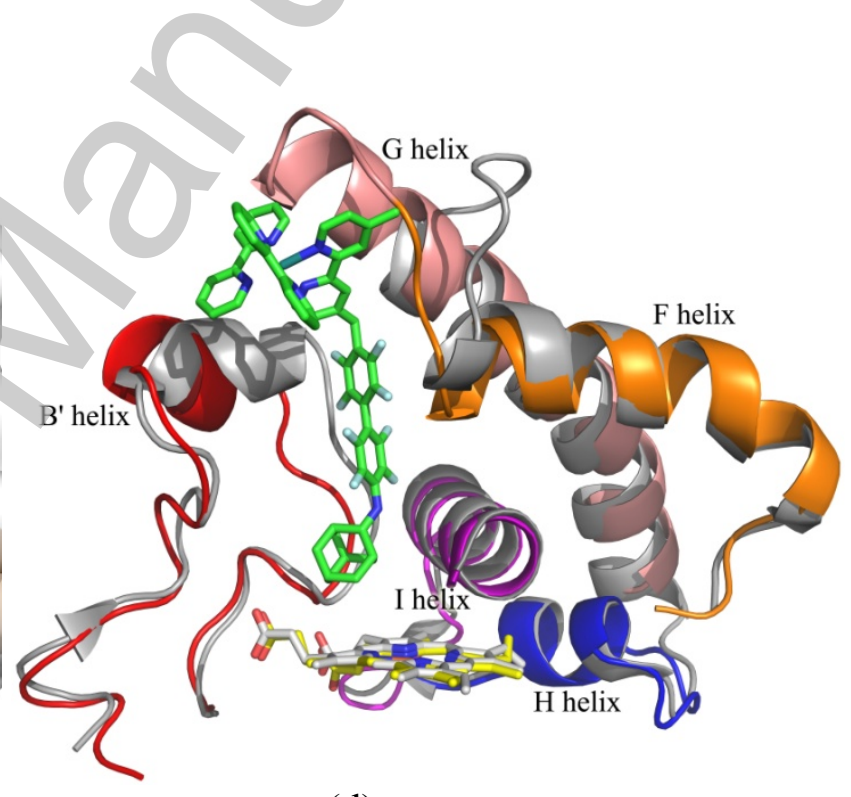

(d)

Figure 1 


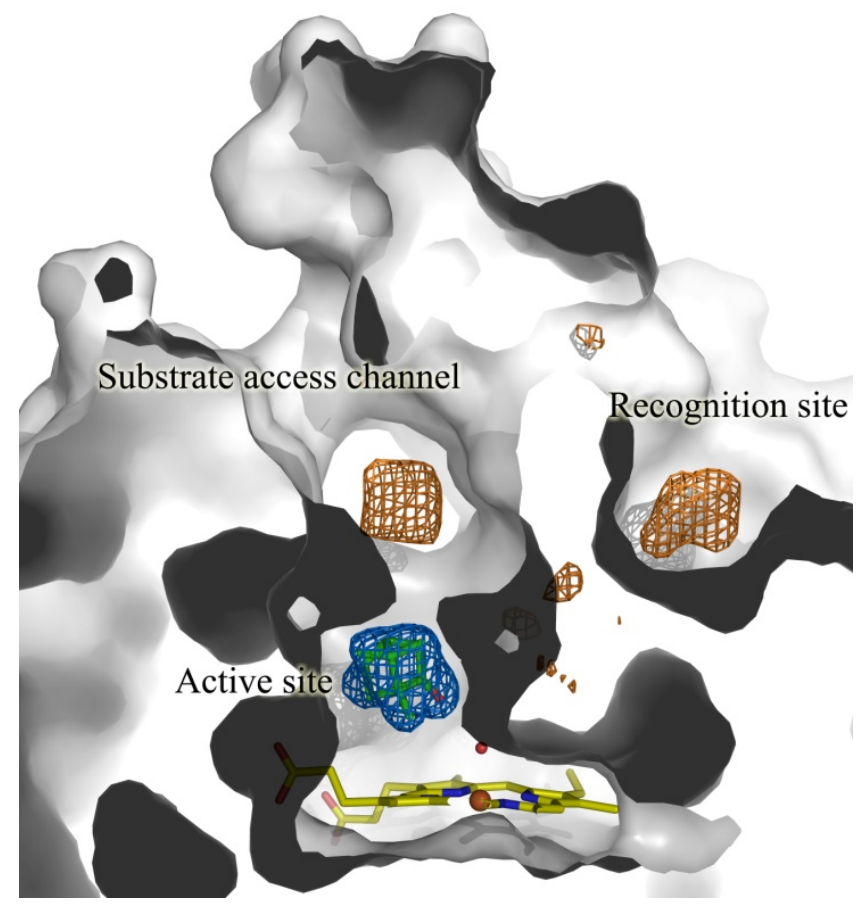

(a)

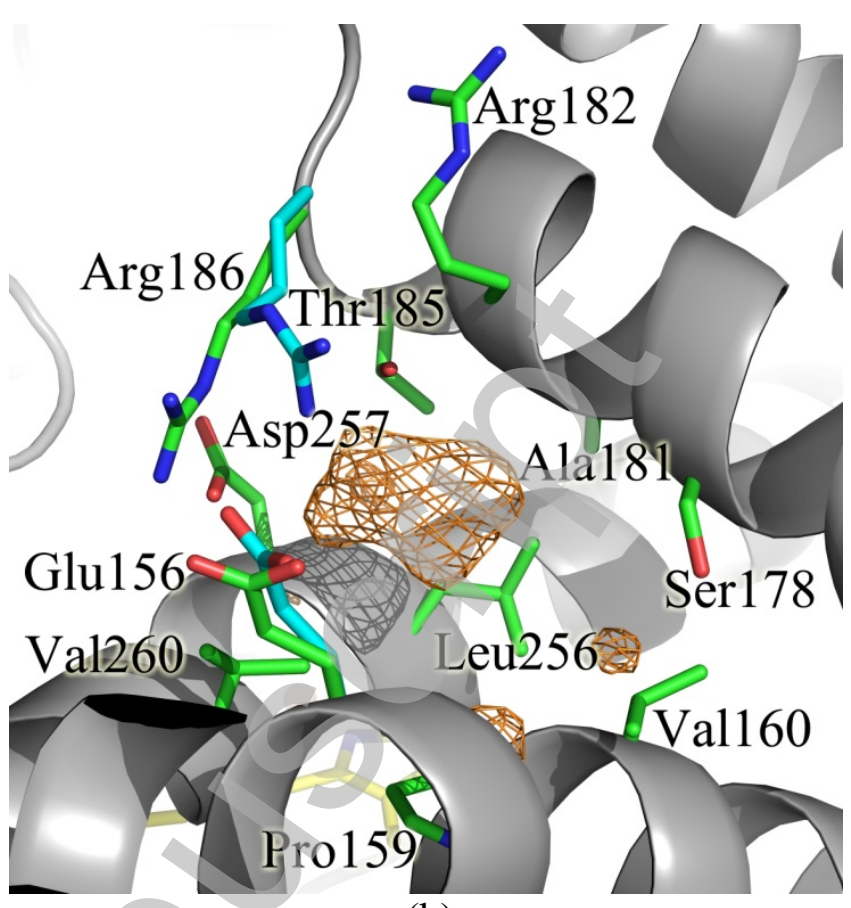

(b)

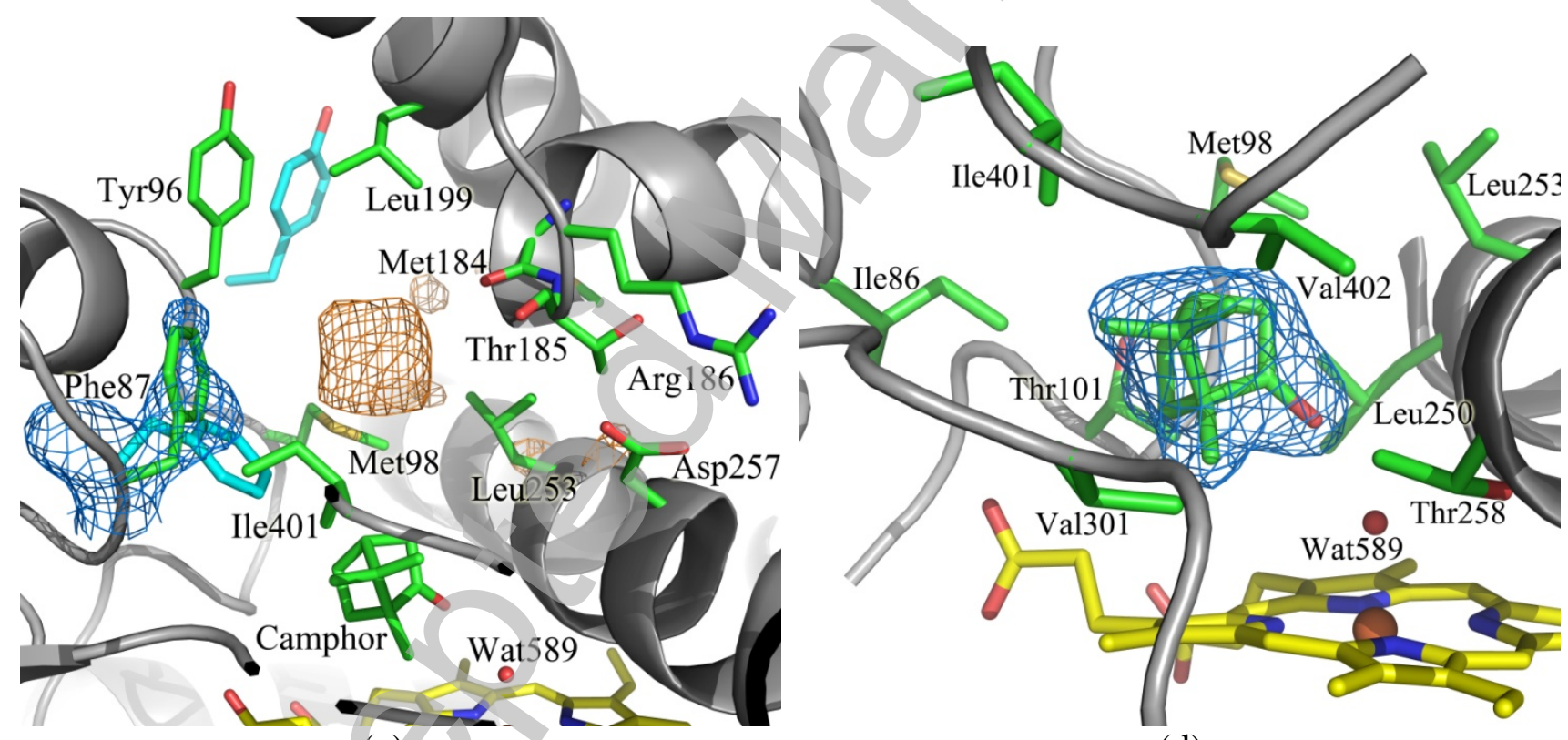

(c)

(d)

Figure 2 


\section{B Biochemical Journal Immediate Publication. Published on 15 Oct 2010 as manuscript BJ20101017}

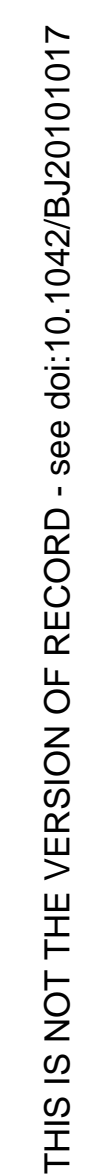

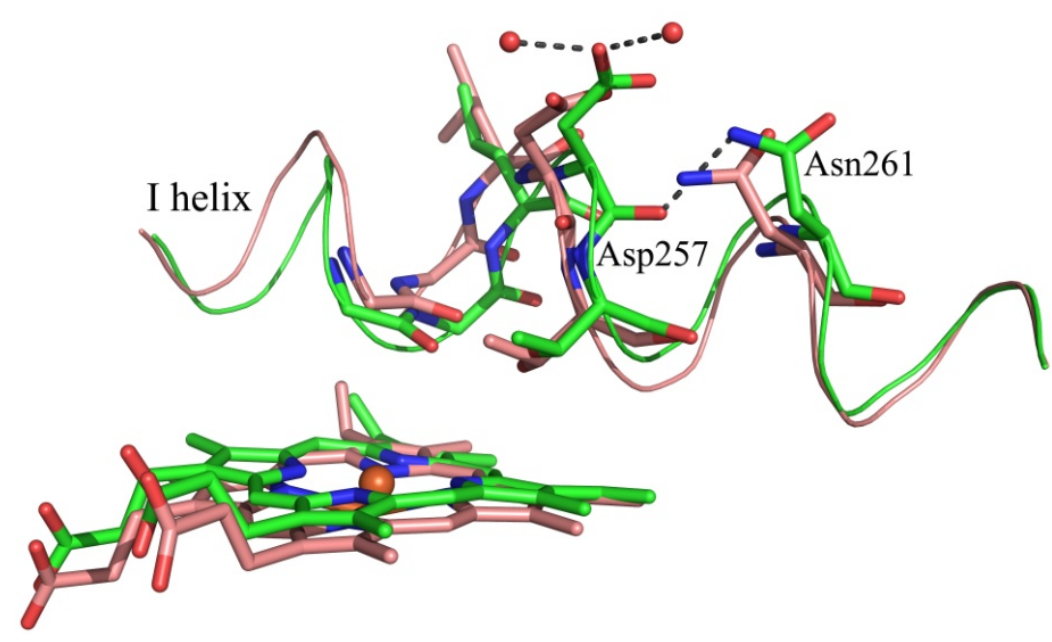

(a)

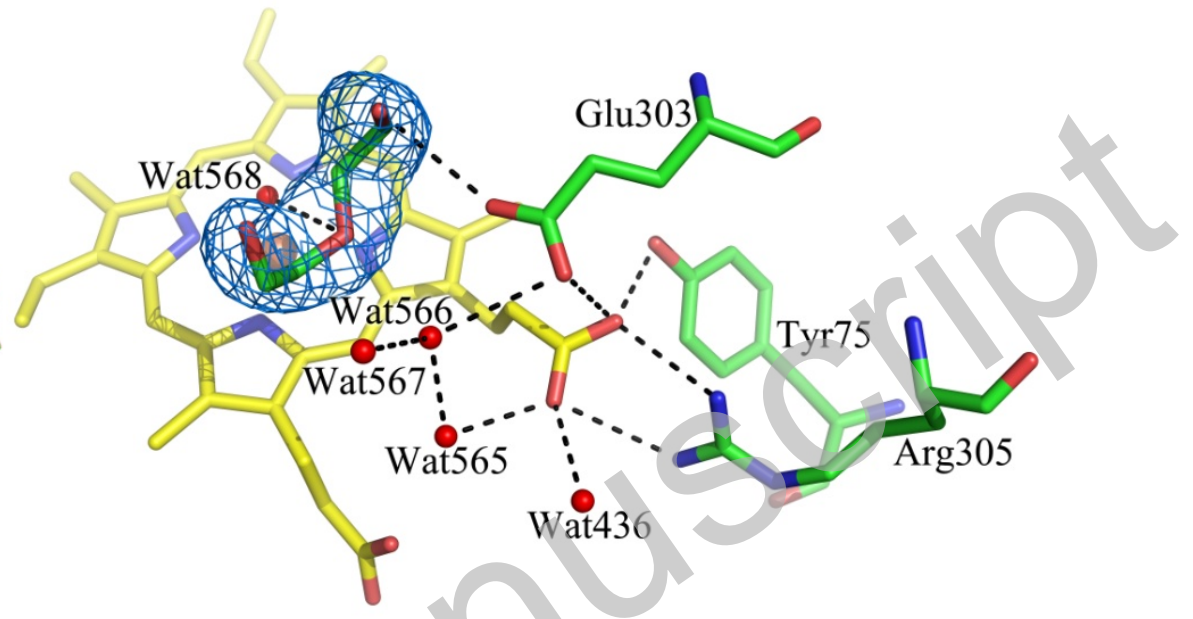

(b)

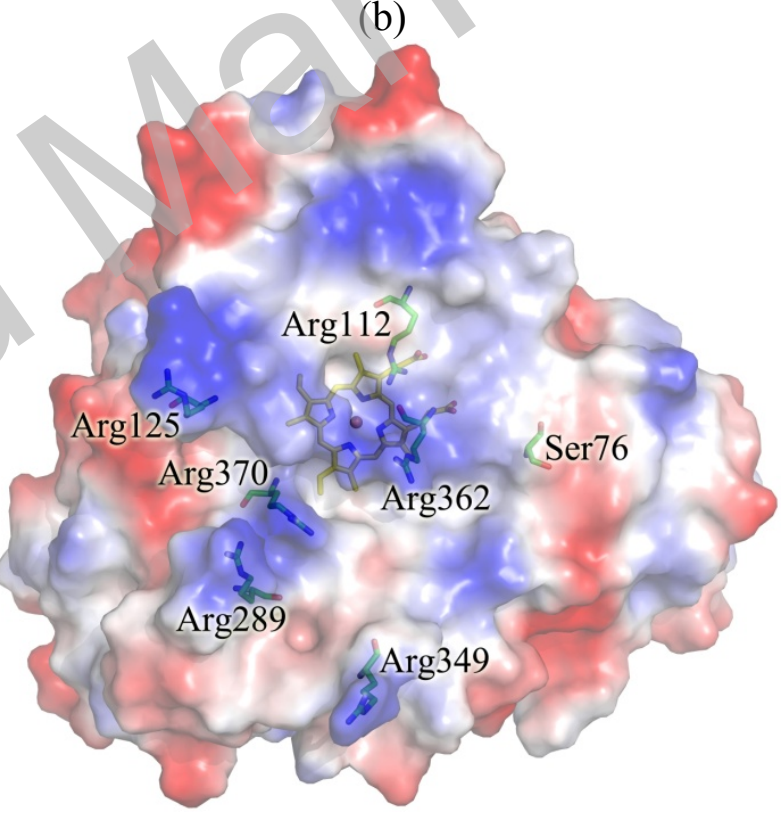

(d)

Figure 3 